\title{
Gata3-deficient mice develop parathyroid abnormalities due to dysregulation of the parathyroid-specific transcription factor Gcm2
}

Irina V. Grigorieva, ${ }^{1}$ Samantha Mirczuk, ${ }^{1}$ Katherine U. Gaynor, ${ }^{1}$ M. Andrew Nesbit, ${ }^{1}$ Elena F. Grigorieva, ${ }^{2}$ Qiaozhi Wei, ${ }^{3}$ Asif Ali, ${ }^{1}$ Rebecca J. Fairclough, ${ }^{1}$ Joanna M. Stacey, ${ }^{1}$ Michael J. Stechman, ${ }^{1}$ Radu Mihai, ${ }^{4}$ Dorota Kurek, ${ }^{5}$ William D. Fraser, ${ }^{6}$ Tertius Hough, ${ }^{7}$ Brian G. Condie, ${ }^{3}$ Nancy Manley, ${ }^{3}$ Frank Grosveld, ${ }^{5}$ and Rajesh V. Thakker ${ }^{1}$

${ }^{1}$ Academic Endocrine Unit, Nuffield Department of Clinical Medicine, University of Oxford, Oxford Centre for Diabetes, Endocrinology and Metabolism (OCDEM), Churchill Hospital, Headington, Oxford, United Kingdom. 2Division of Developmental Neurobiology, The National Institute for Medical Research, The Ridgeway, Mill Hill, London, United Kingdom. ${ }^{3}$ Department of Genetics, University of Georgia, Athens, Georgia, USA. ${ }^{4}$ Nuffield Department of Surgery, University of Oxford, John Radcliffe Hospital, Oxford, United Kingdom. ${ }^{5}$ Department of Cell Biology and Genetics, Faculty of Medicine, Erasmus University Rotterdam, Rotterdam, Netherlands. ${ }^{6}$ Unit of Clinical Chemistry, School of Clinical Sciences, University of Liverpool, Liverpool, United Kingdom. ${ }^{7}$ Clinical Pathology Laboratory, Mary Lyon Centre, Medical Research Council, Harwell, Didcot, Oxfordshire, United Kingdom.

\begin{abstract}
Heterozygous mutations of GATA3, which encodes a dual zinc-finger transcription factor, cause hypoparathyroidism with sensorineural deafness and renal dysplasia. Here, we have investigated the role of GATA3 in parathyroid function by challenging $\mathrm{Gata}^{+/-}$mice with a diet low in calcium and vitamin $\mathrm{D}$ so as to expose any defects in parathyroid function. This led to a higher mortality among Gata3 ${ }^{+/-}$mice compared with $\mathrm{Gata}^{+/+}$ mice. Compared with their wild-type littermates, $\mathrm{Gata3}^{+/-}$mice had lower plasma concentrations of calcium and parathyroid hormone (PTH) and smaller parathyroid glands with a reduced Ki-67 proliferation rate. At E11.5, $\mathrm{Gata}^{+/-}$embryos had smaller parathyroid-thymus primordia with fewer cells expressing the parathyroid-specific gene glial cells missing 2 ( $\mathrm{Gcm} 2$ ), the homolog of human GCMB. In contrast, E11.5 Gata3 ${ }^{-/-}$embryos had no Gcm 2 expression and by E12.5 had gross defects in the third and fourth pharyngeal pouches, including absent parathyroid-thymus primordia. Electrophoretic mobility shift, luciferase reporter, and chromatin immunoprecipitation assays showed that GATA3 binds specifically to a functional double-GATA motif within the GCMB promoter. Thus, GATA3 is critical for the differentiation and survival of parathyroid progenitor cells and, with GCM2/B, forms part of a transcriptional cascade in parathyroid development and function.
\end{abstract}

\section{Introduction}

GATA3 belongs to a family of dual zinc-finger transcription factors that are involved in vertebrate embryonic development, and GATA3 haploinsufficiency has been reported to result in the congenital hypoparathyroidism-deafness-renal dysplasia (HDR) syndrome (1). The mammalian GATA proteins (GATA 1-6) share related Cys- $\mathrm{X}_{2}$-Cys- $\mathrm{X}_{17}$-Cys- $\mathrm{X}_{2}$-Cys (where $\mathrm{X}$ represents any amino acid residue) zinc-finger DNA-binding domains that bind to the consensus motif 5'-(A/T) GATA(A/G)-3' (2). The C-terminal finger $(\mathrm{ZnF} 2)$ is essential for DNA binding, whereas the N-terminal finger $(\mathrm{ZnF} 1)$ helps to stabilize this binding and to physically interact with other proteins such as the multi-type zinc-finger proteins FOG 1 and 2 (3). In vitro studies have shown that the HDR-associated GATA3 mutations result in an impaired binding to the target DNA motif or to a destabilization of this binding or to a lack of interaction with FOG2 $\mathrm{ZnFs}(1,4-6)$; these abnormalities in turn will alter the transcriptional activity of GATA3.

The genes that are regulated by GATA3 in the parathyroids remain largely unknown, while some of the target genes in the kidney and inner ear have been identified by in vitro and in vivo studies of a mouse model that is deleted for Gata3 (7-10). Gata3 $3^{+-}$mice are viable and fertile, but Gata3-- embryos die by E12.5 and have abnormalities of renal morphogenesis $(7,11)$ due to dysregulation of Ret

Conflict of interest: The authors have declared that no conflict of interest exists. Citation for this article: J Clin Invest. 2010;120(6):2144-2155. doi:10.1172/JCI42021. expression, which is important for the development of the mouse kidney and nephric duct (9). Gata3 and Ret have been shown to form part of an important transcriptional cascade, whereby pairedbox (PAX) proteins - PAX2 and PAX8 - participate upstream to activate Gata3 in the renal morphogenesis pathway $(8,9)$. However, it is important to note that the altered renal morphogenesis is observed in $\mathrm{Gata3}^{-/-}$mice but not in Gata3 ${ }^{+/-}$mice, while HDR patients, who are also heterozygous for the GATA3 mutation, have abnormal kidneys (7). In contrast, deafness is found to occur in $\mathrm{Gata3}^{+/-}$mice and is similar to that in HDR patients (12-14). The deafness in $\mathrm{Gata}^{+/-}$mice is due to abnormalities of the inner ear, which include early degeneration of the inner ear hair cells, and part of the underlying mechanism involves abnormalities of the insulin-like growth factor signaling pathway that includes the serine/threonine kinase Akt/PKB $(10,14)$. However, the role of GATA3 in the parathyroid morphogenesis pathway, which includes the transcription factors HOXA3, PAX1, PAX9, TBX1, SOX3, and glial cells missing 2 (GCM2) (15-19), remains to be defined. As a first step, we investigated the development of hypoparathyroidism and parathyroid gland abnormalities in mice deleted for Gata3.

\section{Results}

Parathyroid function in adult Gata3 $3^{+/}$mice. Adult (> 60 day old) Gata $3^{+/-}$ mice maintained on standard chow, which contains $1.15 \%$ calcium and $4.0 \mathrm{iU} / \mathrm{g}$ vitamin $\mathrm{D}$, were found to have plasma calcium concentrations that were similar to those of $\mathrm{Gata}^{+/+}$mice (data not shown). 
A

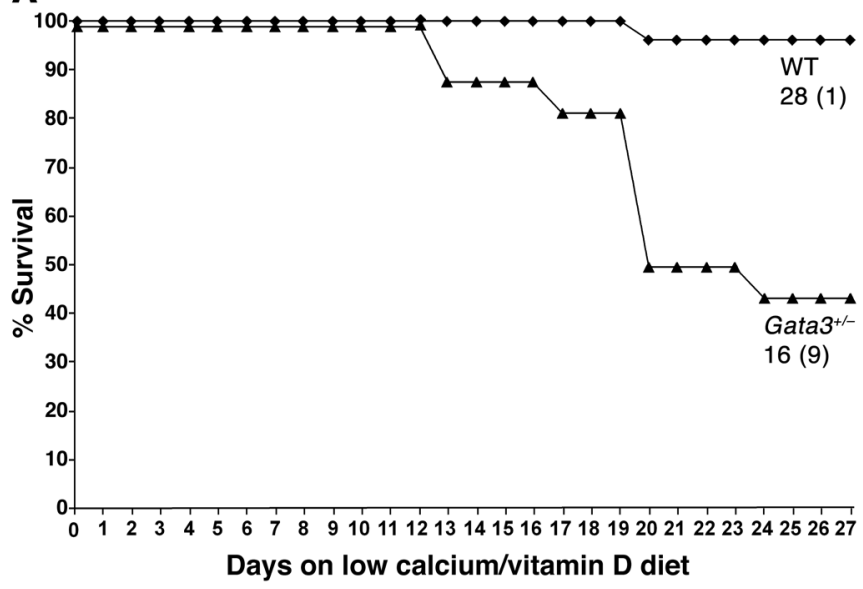

B

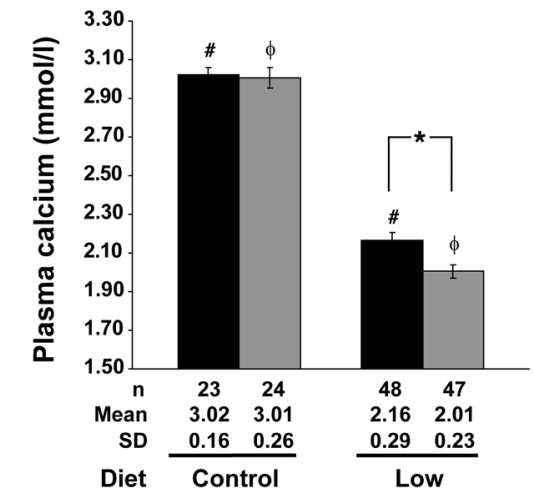

C

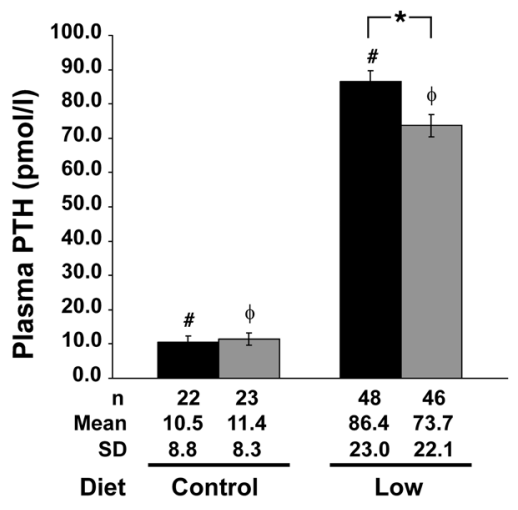

However, this standard chow diet contains amounts of calcium and vitamin $\mathrm{D}$ that are 150 -fold and 30-fold greater, respectively, than the normal daily recommendation intakes in humans. These high dietary contents of calcium and vitamin D may be able to rectify hypocalcemia, and mice were therefore challenged at weaning with a diet low in calcium $(0.001 \%)$ and vitamin $\mathrm{D}(0.0 \mathrm{iU} / \mathrm{g})$. The low calcium/vitamin D diet led to a significantly higher mortality among the $\mathrm{Gata}^{+}{ }^{+-}$mice such that $56 \%$ of $\mathrm{Gata}^{+}{ }^{+-}$mice had died by day 27 , whereas only $3 \%$ of the $\mathrm{Gata}^{+/+}$mice had died $(P<0.001)$ (Figure 1A). The plasma calcium concentrations (adjusted for albumin) as well as those for phosphate and parathyroid hormone (PTH) were similar in the surviving Gata3 $3^{+/}$and $\mathrm{Gata}^{+/+}$mice (data not shown). However, death in these Gata3 ${ }^{+/-}$mice, which was sudden and not

\section{Figure 1}

Effects of low calcium/vitamin D diet on survival and plasma calcium and PTH concentrations. (A) Gata3+/+ (wild-type) and Gata3+/- mice were placed at weaning (day 0) on a low calcium $(0.001 \%) / v i t a m i n ~ D(0.0 \mathrm{iU} / \mathrm{g})$ diet. Survival of Gata3 $^{+/-}$mice on the low calcium/vitamin D diet was significantly reduced. Sudden death occurred in $\mathrm{Gata3}^{+/-}$mice after 13 days on the low calcium/vitamin D diet. By day 27, there were less than $50 \% \mathrm{Gata}^{+/-}$mice surviving, and the study was terminated. In contrast, more than $95 \%$ of the Gata3 ${ }^{+/+}$mice survived $(P<0.001)$. Numbers of mice in each group are indicated on the right, and number of deaths are shown in parentheses. (B) Plasma-adjusted calcium and PTH concentrations were assessed 12 days after weaning while on control $(0.95 \%$ calcium and $4.5 \mathrm{iU} / \mathrm{g}$ vitamin D) or low calcium/vitamin D diets. Gata3 $3^{+/+}$ and Gata3 $^{+/-}$mice had similar plasma calcium concentrations on the control diet but became significantly hypocalcemic on the low calcium/ vitamin D diet, although the Gata3 ${ }^{+/}$mice had significantly lower plasma calcium concentrations than their Gata3+/+ littermates. (C) Gata3 ${ }^{+/+}$and $\mathrm{Gata3}^{+/-}$mice had similar plasma PTH concentrations on the control diet, and this rose significantly in response to the low calcium/vitamin D diet, although the elevation in plasma PTH was less in the Gata3 ${ }^{+/-}$mice. Gata3 $^{+/+}$(black bars); Gata3+/- (gray bars). Error bars represent SEM. $\# P<0.000001$, Gata3 ${ }^{+/+}$mice on control versus low diet; $\phi P<0.000001$, Gata3 $^{+/-}$mice on control versus low diet; ${ }^{*} P<0.01$.

observed to be associated with seizures or neuromuscular irritability, occurred from day 13 on the low calcium/vitamin D diet, suggesting that these mice may have been developing hypocalcemia prior to this time. Plasma samples were therefore collected on day 12 after starting the low calcium/vitamin D or control diet. Gata3 $3^{+/+}$and Gata3 ${ }^{+/-}$mice on a control or a low calcium/vitamin D diet had similar plasma sodium, potassium, urea, creatinine, chloride, total protein, albumin concentrations, and alkaline phosphatase activity (data not shown). Moreover, the mean plasma calcium and PTH concentrations in the $\mathrm{Gata3}^{+/+}$and $\mathrm{Gata3}^{+/-}$mice on the control diet were not significantly different. However, in the Gata3 ${ }^{+/}$and $\mathrm{Gata}^{+/-}$mice on the low calcium/vitamin D diet, the mean plasma calcium concentrations were lower, and the mean circulating PTH concentrations were higher; furthermore, the $\mathrm{Gata}^{+/-}$mice had significantly lower mean plasma calcium concentrations than the Gata $3^{+/+}$mice $(2.01 \pm 0.23$ versus $2.16 \pm 0.29 \mathrm{mmol} / \mathrm{l} ; P<0.01)$, but this was associated with lower mean PTH concentrations $(73.7 \pm 22.1$ versus $86.4 \pm 23.0 \mathrm{pmol} / \mathrm{l}$; $P<0.01$ ) (Figure 1, B and C). These results suggested a defect in PTH secretion in response to hypocalcemia in the Gata $3^{+/}$mice.

Parathyroid gland size and proliferation in adult $\mathrm{Gata}^{+/-}$mice. The significantly lower plasma calcium concentrations observed in the $\mathrm{Gata3}^{+/-}$mice on the low calcium/vitamin D diet and a lower circulating PTH concentration than that observed in the $\mathrm{Gata}^{+/+}$mice (Figure 1, B and C) suggested a blunted PTH secretory response in the $\mathrm{Gata}^{+/-}$mice. PTH secretion has been reported to correlate with parathyroid gland size (20), and the size of the parathyroid glands in the $\mathrm{Gata}^{+/-}$and $\mathrm{Gata}_{3}^{++/}$mice were therefore assessed (Figure 2A). Cross-sectional areas were measured using H\&Estained sections (21) and corrected for body weight. Gata $3^{+/+}$and $\mathrm{Gata3}^{+/-}$mice on the control diet had similar parathyroid gland sizes. However, on the low calcium/vitamin D diet, the parathyroid gland sizes of the Gata3+/, but not of the $\mathrm{Gata}^{+/-}$, mice were significantly increased (approximately 2 -fold, $P<0.05$; Figure 2A). Thus, the parathyroids of the Gata3 ${ }^{+/+}$mice became significantly larger than those of the $\mathrm{Gata}^{+/-}$mice. This enlargement of parathyroid gland size was due to increased proliferation (Figure 2B), as demonstrated by the increase in the mean Ki-67 proliferation rate. In 
A
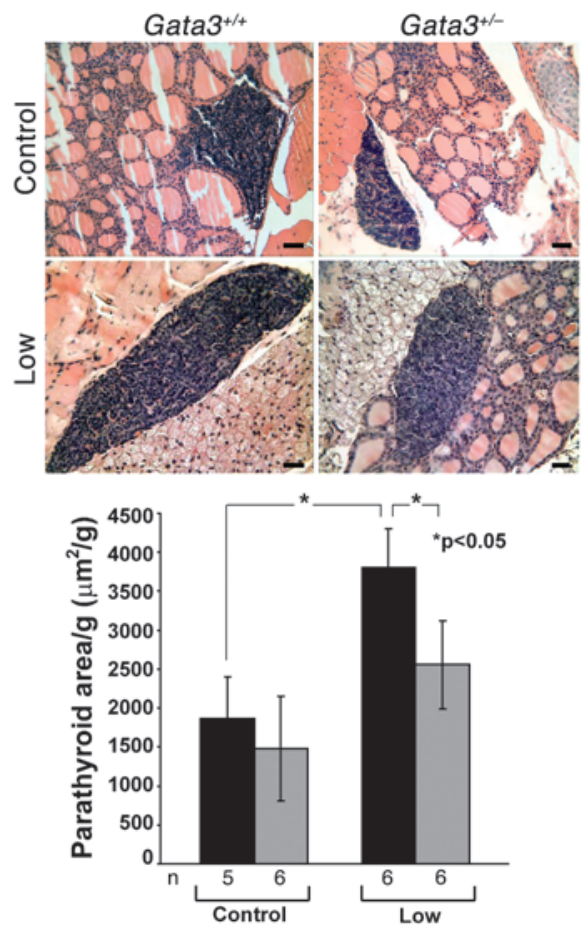

B
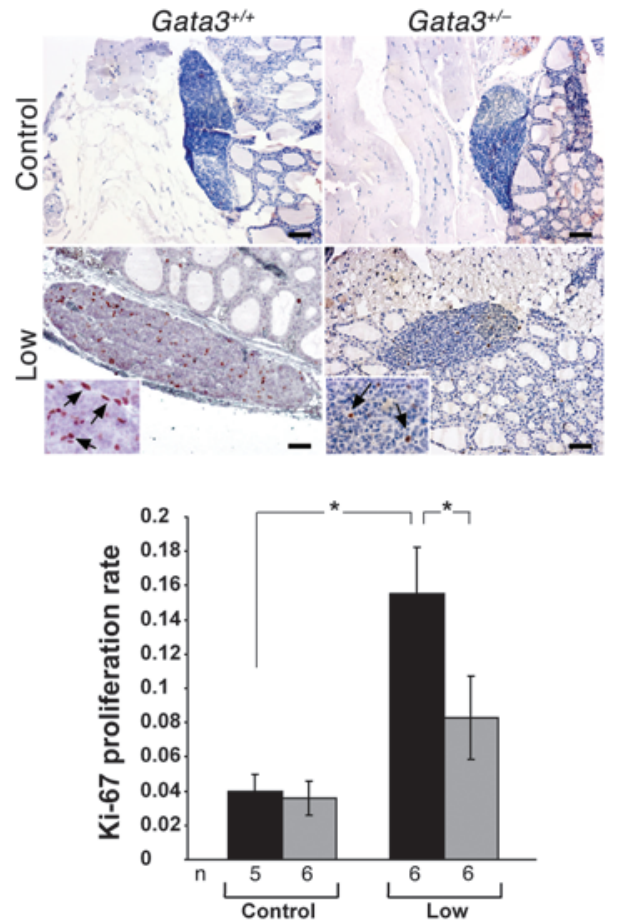
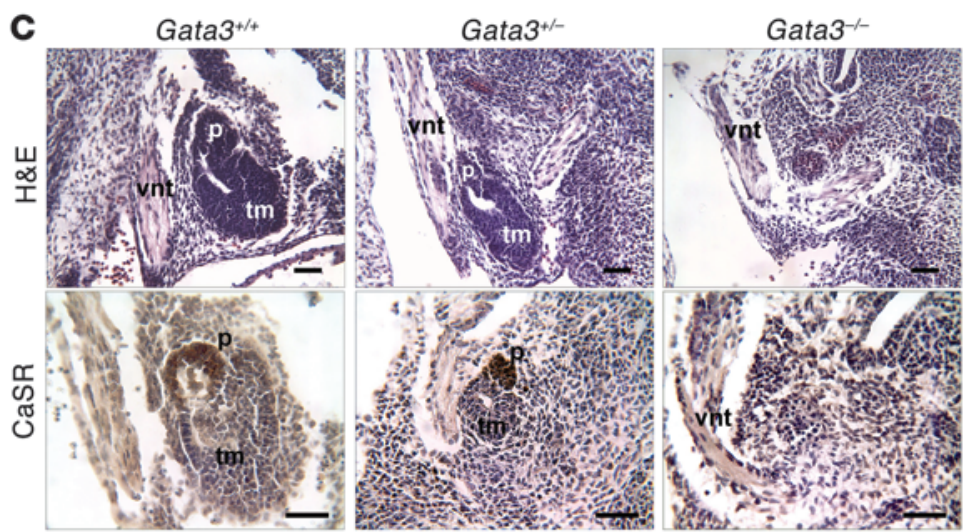

D

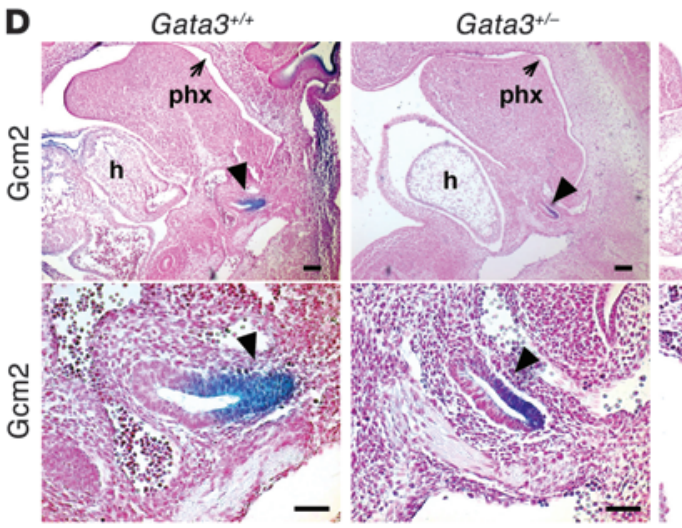

Gata3--

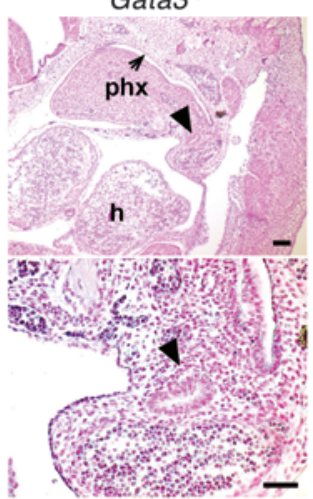

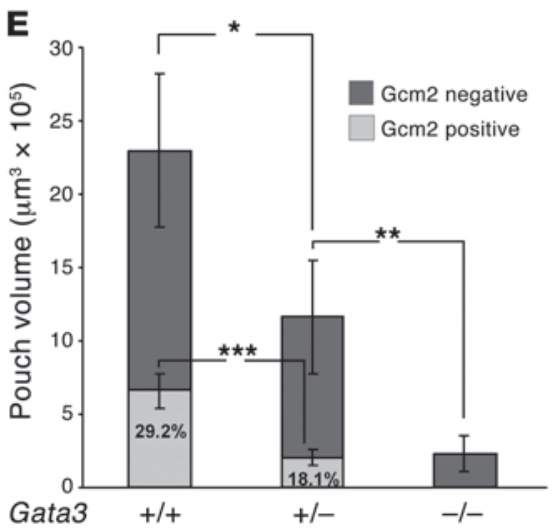




\section{Figure 2}

Parathyroid histology and proliferation studies in Gata3+/- and $\mathrm{Gata3}^{-/-}$ mice. (A) Parathyroid size in Gata3 ${ }^{+/+}$and Gata3 $3^{+/}$mice after 12 days, after weaning, on control or low calcium/vitamin D diets. H\&Estained parathyroid sections (top). Scale bars: $50 \mu \mathrm{m}$. Parathyroid size assessed by cross-sectional area analysis of longitudinally cut serial sections (6 microns) from 5-6 animals per group $(n)$ and corrected for body weight $\left(\mu \mathrm{m}^{2} / \mathrm{g}\right)$ in $\mathrm{Gata}^{+/+}$(black bars) and Gata3 ${ }^{+/-}$(gray bars) mice. (B) Parathyroid proliferation rates assessed by Ki- 67 immunostaining. Nuclear Ki-67 immunostaining in parathyroid cells (top). Scale bars: $50 \mu \mathrm{m}$. Insets show details at higher magnification $(\times 100)$, and arrows point to nuclear staining. Ki-67 proliferation rate (Ki-67-positive cells/total number of cells) in Gata3 ${ }^{+/+}$(black bars) and Gata3 $^{+/-}$(gray bars) mice. ${ }^{*} P<0.05$. (C) Studies of parathyroid-thymus primordia in E12.5 embryos. H\&E staining (top panel) and corresponding serial section immunostained with CaSR antibody (bottom panel). Scale bars: $50 \mu \mathrm{m}$. $P$, parathyroid; tm, thymus; vnt, vagus nerve trunk. (D) Sagittal sections of pharyngeal pouches and pharynx (phx) from E11.5 embryos hybridized with Gcm2 riboprobe to identify the parathyroid domain of the common parathyroid-thymus primordium (arrows) arising from the third pharyngeal pouch. Scale bars: $100 \mu \mathrm{m}$ (top panels); $50 \mu \mathrm{m}$ (bottom panels). $\mathrm{h}$, heart. (E) Volume of third pharyngeal pouch in E11.5 embryos ( $n=6$ of each genotype) and the proportion of Gcm2-expressing cells. ${ }^{\star} P<0.02 ;{ }^{* \star} P<0.001 ;{ }^{* *} P<0.002$. $P$ values calculated using Student's $t$ test. Error bars represent SEM.

the parathyroids of mice on the low calcium/vitamin D diet, the increase was approximately 4 -fold $(P<0.05)$ in the $\mathrm{Gata}^{+/+}$mice and only 2 -fold in the Gata3 $3^{+/-}$mice when compared with those on the control diet. Furthermore, the mean proliferation rate of parathyroids in the $\mathrm{Gata3}^{+/-}$mice on the low calcium/vitamin D diet was significantly $(P<0.05)$ lower than that of the $\mathrm{Gata3}^{+/+}$ mice. The rates of apoptosis in the parathyroids of the $\mathrm{Gata3}^{+/-}$and Gata3 $^{+/+}$mice on the control or low calcium/vitamin D diets were less than $0.001 \%$ and not significantly different (data not shown). These findings indicate that the diminished parathyroid gland enlargement in the $\mathrm{Gata3}^{+/-}$mice may be caused by a lower proliferation rate and not an increased apoptosis rate, and we therefore investigated $\mathrm{Gata3}^{+/+}$, $\mathrm{Gata3}^{+/-}$, and $\mathrm{Gata3}^{-/-}$embryos for abnormalities of parathyroid proliferation and differentiation.

Role of Gata3 in developing parathyroid primordia. We used E12.5 and E11.5 mouse embryos to study the role of Gata3 in parathyroid cell proliferation and differentiation because it is at these stages that parathyroid development becomes distinguishable from the third pharyngeal pouches. The third pharyngeal pouches become visible by E9.5-E10, and by E11-E11.5 epithelial outgrowths form the bilateral parathyroid-thymus primordia that consist of a dorsalanterior parathyroid domain, which specifically expresses $G \mathrm{~cm} 2$, and a ventral-posterior thymus domain that expresses forkhead box n1 (Foxn1) (17, 22-24). Each primordium then separates into one parathyroid gland and one thymus lobe at E12.5-E13.5, which then migrate to their eventual adult locations by about E14.5 $(25,26)$. To identify parathyroid cells, we used expression of the calciumsensing receptor (CaSR) in E12.5 embryos (17) and expression of Gcm2 in E11.5 embryos (22). Investigation of the expression patterns of Gata3 and Gcm 2 revealed them to be overlapping in the pharyngeal endoderm of developing Gata $3^{+/+}$mouse embryos, with Gata3 expression occurring from E8.5 and localizing in the parathyroid domain of the common parathyroid-thymus primordium at E11.5 (Supplemental Figure 1; supplemental material available online with this article; doi:10.1172/JCI42021DS1). Gata3 ${ }^{+/-}$E12.5 embryos were found to have smaller parathyroid-thymus primor- dia that contained fewer CaSR immunostaining cells (Figure 2C). In addition, Gata3 ${ }^{-/-}$E12.5 embryos had gross developmental defects of the third and fourth pharyngeal pouches that included absent parathyroid-thymus primordia evident by examination of H\&E-stained serial sections and by the lack of CaSR in the third pouch (Figure 2C). These abnormalities of primordia size in $\mathrm{Gata3}^{+/-}$and $\mathrm{Gata3}^{-/-}$embryos were also present earlier in E11.5 embryos (Figure 2D). The volume of the third pouch, which was estimated using serial sections (27), was 50\% smaller in $\mathrm{Gata3}^{+/-}$ E11.5 embryos when compared with that of Gata3 ${ }^{+/+}$embryos (Figure 2E), consistent with the observed size differences at E12.5. Moreover, Gata3 ${ }^{-/-}$E11.5 embryos were found to have a very small third pouch in association with severe abnormalities of the pharyngeal region (Figure 2D). This indicates that although the formation of the parathyroid-thymus primordia is initiated in E11.5 Gata3 $^{-/}$embryos, it does not progress further and by E12.5, these primordia are lost. This lack of further differentiation into parathyroid primordia is likely due to the observed absence of $\mathrm{Gcm} 2$ expression in cells of the third pouch in $\mathrm{Gata3}^{-/-}$embryos, and the smaller parathyroid size in the $\mathrm{Gata3}^{+/-}$embryos is likely due to the markedly reduced proliferation of Gcm2-expressing cells in the third pouch when compared with Gata3 $^{+/+}$embryos $(18.1 \% \pm 3.6 \%$ versus $29.2 \% \pm 1.8 \%$ of the total third pouch volume, $P<0.002$; Figure 2E). Thus, Gata3 ${ }^{+/}$embryos have smaller third pharyngeal pouches as well as an approximate $40 \%$ reduction in the proportion of cells that are specified to the parathyroid fate as defined by Gcm 2 expression. These results indicate that Gata3 and Gcm 2 may form part of a transcriptional cascade that determines parathyroid gland development and parathyroid cell proliferation. This would be consistent with the reports that ablation of Gcm2 in mice leads to complete loss of parathyroid glands (28), while deletions and mutations of the GCMB gene, which is the human homolog, result in isolated hypoparathyroidism $(29,30)$, while GCMB overexpression is associated with parathyroid tumors (31).

Putative GATA3-binding sites in the GCMB promoter. On the basis of Gata3 and Gcm 2 coexpression in the third pharyngeal pouch of wild-type E11.5 mouse embryos (Supplemental Figure 1) and the absence of $\mathrm{Gcm} 2$ expression in the Gata3 $3^{-/}$embryos together with the marked reduction in Gcm 2 expression in the $\mathrm{Gata}^{+/-}$embryos (Figure 2, D and E), we postulated that GATA3 may directly regulate the expression of $G c m 2 / G C M B$. Gcm $2 / G C M B$ is specifically expressed in the parathyroid glands $(22,28)$, which weigh less than $0.125 \mathrm{mg}$ in adult mice (21), thereby making it difficult to obtain sufficient quantities of RNA and chromatin to investigate $\mathrm{Gcm} 2$ transcripts and binding to the promoter region in mouse parathyroids. However, in humans, the parathyroid glands weigh $90-130 \mathrm{mg}$ (32) and parathyroid adenomas, which express GCMB (31), may weigh from $100 \mathrm{mg}$ to several grams. We therefore used human parathyroid adenomas to obtain sufficient quantity of RNA and chromatin for investigation of the proximal promoter region of the human GCMB gene for functionally active GATA3-binding sites. The human GCMB gene, which is located on chromosome 6p23, has been reported to consist of 5 exons with the ATG translation start codon in exon 1 and a transcription start site residing approximately 70 bp upstream of the ATG codon, as determined by analysis of the longest clone isolated from a human fetal brain cDNA library (33) (Figure 3A). To characterize further the promoter region of GCMB, which is specifically expressed in parathyroid glands, we used total RNA from parathyroid adenomas for 5' rapid amplification of cDNA ends (RACE) (Figure 3, B and C). DNA sequence 

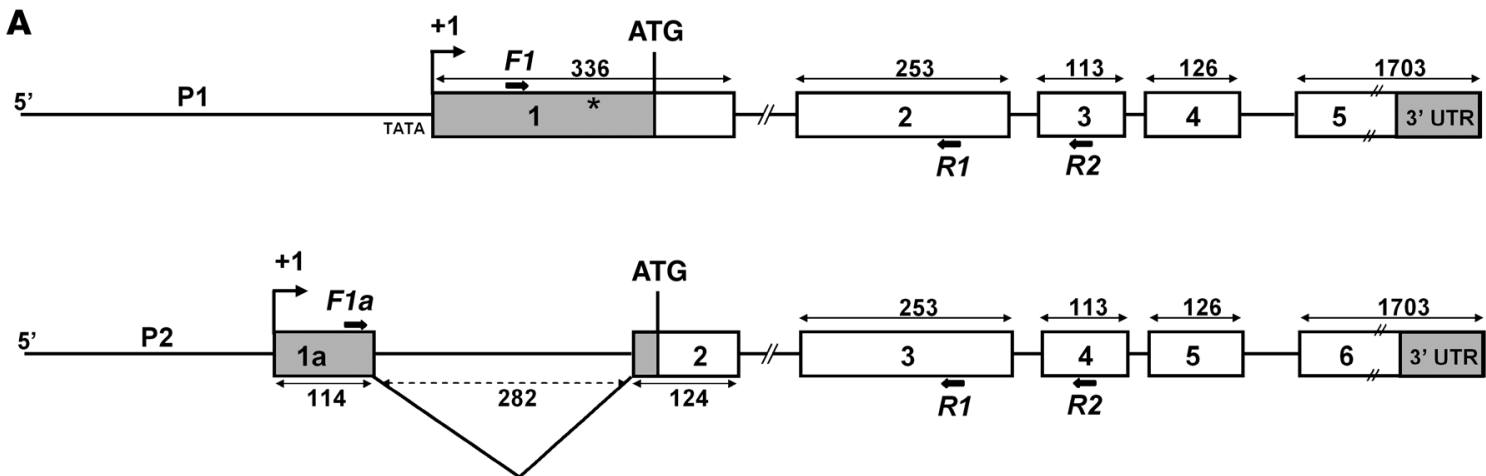

B Common Reverse primer:

Specific Forward primer:

$\frac{R 1}{\frac{F 1}{n \quad 1 / 20} \frac{F 1 a}{n \quad 1 / 20}} \frac{F 1}{n \quad 1 / 20} \frac{F 1 a}{n \quad 1 / 20}$

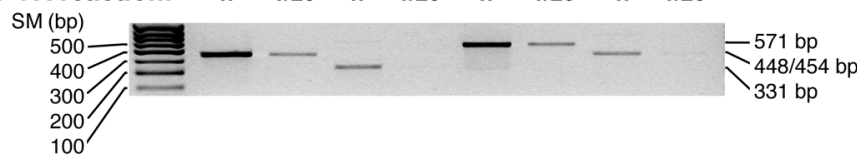

C

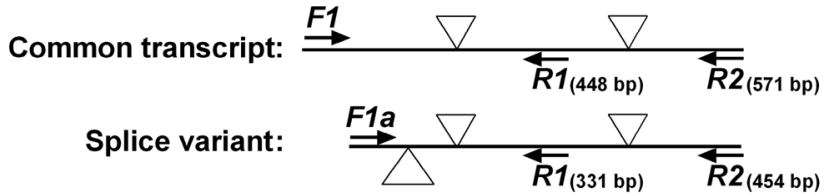

D -1400 tttgagaggccgagacaggaggatcacgaagtcaaaaaatcgagaccatcctggccaacttggtaaaaccctgtctctactaacaatacaaaattagctg

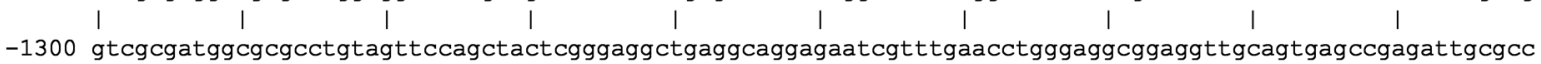

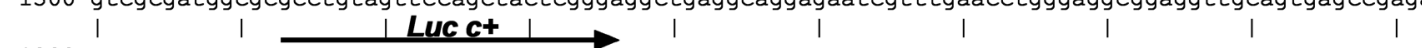

-1200 actgcactccagccgggaagacagagtgagactccgtctcaaaaaaaaaaaaaaaaaaagatttgtgttcgggtgggtggcaactgagagctcgccga

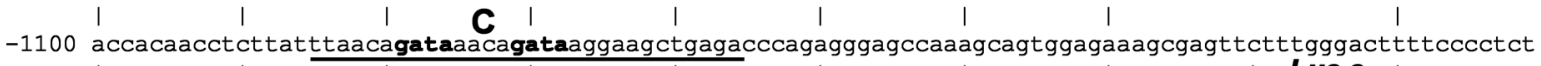

-1100 accacaacctcttatttaacagataacagataaggaagctgagacccagagggagccaaagcagtggagaaagcgagttctttgggacttttccctct

-1000 cccetttccetccttcccaggctcccacctaccccaggctcccacctaccccgggctcatttccgcccccacctcctccaggttcactttgtaggact

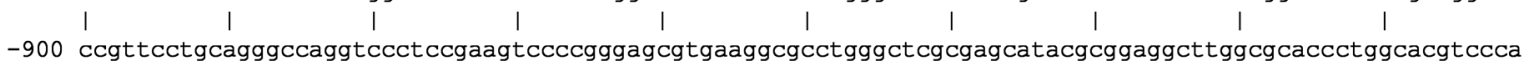

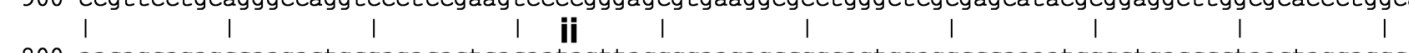

-800 aacagcagagccaagactgcgagacactcacaatagttaggggaagagccggcagtggaggcccaaaatgggctgacccctaactaggaggcgtgggcgc

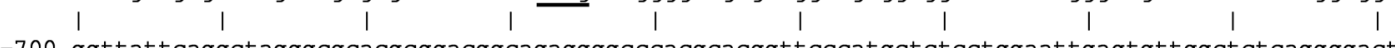

-700 ggttattcaggctagggcgcacgcggacggcagaggggcccacgcacggttcccatgctctcctggaattgagtgttggctctcaggggactttgctgga

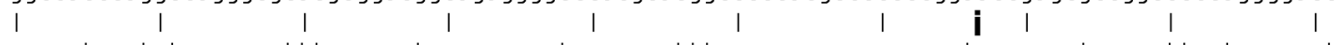

-600 aagcctccatctaagccctttccgaaatcagggaagctggacgcgtttagaaaaaagaaaaaaataggccggtcgcggttgctcacgcctgtaatccc

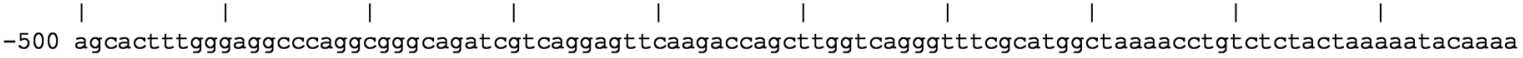

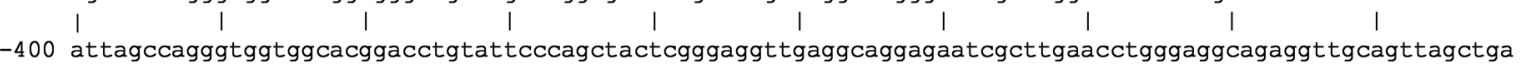

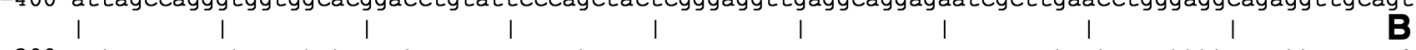

-300 gatggcgccactgcactctagctgggcaacagagtgagacaaaaaagaagaaaagaaaagaagaaaataataaggttttaaacttaaaggatacattcc

-200 tacttaccctctCCaCCCCCACCCTCTGGCTGCAGTITCCATPTGCTICCAGGGCTGAGTIPAACCTCCACCTCCGTTCCTGGGACTGTGGTGGAGGGGG

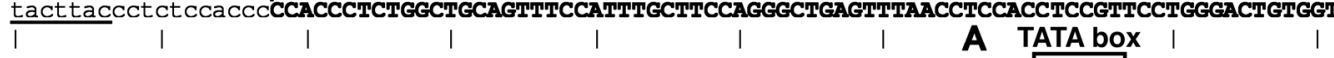

-100 CGACCGGCCCCTGGAGCGTGGGCGCTAGCGAGgtaggCgagacagggcggagcCCCCCggaggtatatet tataaajgagggggcagctgccagaggtC

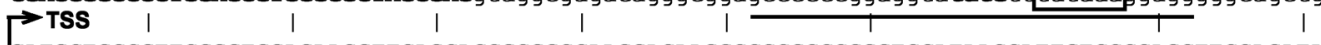

+1 GATGCTGCCGTTCGGGTGGAGAACGTTCAGCACCGCGGCCAAGGACAAGGCCCCGCCCGGGTCCATAACCATTGTCCCCAGCTTCCAGATATCTAAACAG

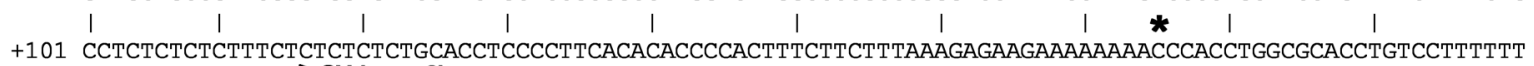
| | $\quad$ | SV (exOn 2) | GCMB RX | $\quad$ |

+201 CTTCTATTTTTCAGAACCCTGGGCGGAAAGCGCGAGCGAGTTGGGCAGATGCCGGCGGCCGCGGTGCAGGAAGCGGTCGGCGTGTGCTCCTACGGGATGC

+301 AGCTCAGCTGGGACATCAACGATCCGCAGATGCCTCAG 338 \begin{tabular}{lllllllllllllllllll}
\hline$Q$ & $L$ & $S$ & $W$ & $D$ & $I$ & $N$ & $D$ & $P$ & $Q$ & $M$ & $P$ & $Q$ & $\ldots$ & $\ldots$
\end{tabular} 


\section{Figure 3}

Mapping of the GCMB transcription start sites by 5 ' RACE. (A) Representation of human GCMB gene showing coding exons (white boxes) and 2 alternatively transcribed first exons ( 1 and $1 \mathrm{a}$ ), which contain $5^{\prime}$ UTRs (gray boxes). Transcription start sites are marked +1 and represent the longest 5' RACE amplicons. Primer sequences (arrows; forward, F1 and F1a; and reverse, R1 and R2) used for amplification of exon 1a and exon 1 are shown. (B) Detection of transcript-specific PCR products after 20 cycles of amplification using human parathyroid tumor RNA, at 2 concentrations ( $n$, neat; 1/20, 20-fold dilution of neat). The splice variant has a shorter exon 1 and yields products of $331 \mathrm{bp}$ and $454 \mathrm{bp}$. (C) Representation of primer positions and expected sizes of transcripts. (D) Human GCMB 5' upstream sequence. Locations of previously reported (32) transcription start site $\left(^{*}\right)$ isolated from human fetal brain and one identified (TSS and +1 ) from human parathyroids by this study are indicated. An additional transcription start site that was also identified in a splice variant (SV) is shown. Putative GATA3-binding sites $(\mathbf{A}-\mathbf{C})$ are in bold; GATA motifs in the reverse orientation are indicated as $\mathrm{i}$ and ii; oligonucleotide sequences used for EMSAs are solid underlines; forward primers (Luc-C $\mathrm{C}^{+}$and Luc-C-) used for the luciferase reporter constructs are indicated by arrows; GCMB-RX primer is indicated by the broken underline; and putative TATA box is boxed.

analysis of the GCMB transcript clones revealed the presence of a transcript with a longer $5^{\prime}$ UTR, in which the transcription start site (+1) was 249 bp from the ATG codon (Figure 3, A, B, and D). This finding is in agreement with a report (34) published during the course of this study, in which 5' RACE of RNA extracted from a human parathyroid adenoma revealed that the transcription start site was located 248 bp upstream of the ATG in exon 1 (Figure 3D). However, our study also identified another transcript that started further upstream and contained an alternative exon of $114 \mathrm{bp}$ that spliced to an acceptor splice site, which we believe to be novel, some 34 bp from the ATG site (Figure 3, A, B, and D). Thus, this alternative transcript contains 6 exons, in which the first exon (exon 1a) is noncoding (Figure 3A). Semi-quantitative PCR was used to determine the abundance of each transcript in the parathyroids. This revealed that the amount of the splice variant containing exon $1 \mathrm{a}$ was equivalent to a 20 -fold dilution of the transcript containing exon 1, thereby indicating that this splice variant is the minor transcript that contributes approximately $5 \%$ to the total GCMB RNA pool in parathyroids.

We analyzed the promoter region of the more abundant GCMB transcript, which has the ATG codon in exon 1, for putative GATA3-binding sites in the 1.4-kb DNA sequence that is $5^{\prime}$ to the +1 transcription start site (TSS) (Figure 3D). The promoter region of the transcript contains a canonical TATA box at approximately $-30 \mathrm{bp}$ from the TSS (Figure 3D). GATA proteins recognize canonical 5'-(A/T)GATA(A/G)-3' DNA binding sites as well as others that conform to the sequence 5'-(A/T/C)GAT(A/T)(A)-3' (35). Analysis of the 1.4-kb sequence for these motifs revealed 3 putative GATA3-binding sites, A to $C$ (Figures $3 \mathrm{D}$ and $4 \mathrm{~A})$. Among these, site $\mathrm{C}$ contained a double-GATA motif at position -1082 bp to -1066 bp from the transcription start site $(+1)$ (Figure 3D). An examination of the approximately $1.5-\mathrm{kb}$ genomic region of the mouse $\mathrm{Gcm} 2$ gene revealed that it also contained a double-GATA motif as well as 6 other putative GATA3-binding sites (Supplemental Figure 2). All 3 of the GCMB GATA sites with the core GAT motif were functionally characterized using EMSAs and luciferase reporter assays. Two other sites with a reverse orientation GATA motif ( $5^{\prime}$-ATAG-3', i and ii; Figure 3D) were used as negative controls to assess for nonspecific binding and activity.
Binding by GATA3 protein to GATA motifs in the GCMB promoter region. To determine whether the 3 GATA motifs could physically interact with the GATA3 protein, EMSAs were performed as described (4) using nuclear extracts from COS-7 cells transfected with a wild-type GATA3 construct prepared in pcDNA3.1 (GATA3-pcDNA3.1) and a ${ }^{32} \mathrm{P}-$ labeled double-stranded approximately 30-bp oligonucleotide that contained a GATA motif from the endogenous GCMB promoter (Figure 4A). Quantification of the proportion of bound oligonucleotide relative to the total oligonucleotide (i.e., bound and unbound) was carried out by densitometry to yield an approximation of the strength of binding. This revealed that the double-GATA site $\mathrm{C}$ showed strong binding (Figure 4B) in which approximately $93 \%$ of the oligonucleotide was bound to GATA3 protein (Figure 4B), which was confirmed using a super-shift assay with a GATA3 antibody (Figure 4C). This site is located at position $-1082 \mathrm{bp}$ to $-1066 \mathrm{bp}$ (site C) from the transcription start site $(+1)$ (Figures $3 \mathrm{D}$ and $4 \mathrm{~A})$. This strong binding is consistent with a report that GATA proteins form dimers on DNA with double-GATA sites by an association of the N-and C-terminal zinc-fingers of separate molecules (36). Sites A and B showed only weak binding to GATA3 protein, while the negative controls ( $\mathrm{i}$ and $\mathrm{ii}$ ) showed no binding to GATA3 protein (Figure 4B). Thus, these EMSA studies indicate that site C, with its double-GATA motif, is likely to be the functionally significant GATA3-binding site (Figure 4A).

Characterization of GATA motifs in the promoter region of GCMB using luciferase reporter assays. Cell lines derived from normal parathyroids of humans or any other species were not available, and other cell lines were therefore assessed for the expression of parathyroidspecific genes to facilitate the selection of an appropriate one in which to perform luciferase reporter assays. RNA extracted from the cell lines COS-1 and COS-7 (African green monkey kidney fibroblast-like cell lines); HEK-293 (human embryonic kidney); HK-2 and HKC-8 (human proximal tubule cells derived from normal kidney cortex); human parathyroid adenoma and kidney was used with exon-specific primers to detect expression of GATA3, FOG2, GCMB, PTH, and CASR by RT-PCR. All of these transcripts were expressed in the parathyroid adenoma (Figure 5A), and all but PTH and GCMB were expressed in the kidney, indicating the parathyroid specificity of PTH and GCMB expression. However, both GATA3 and FOG2 were expressed in the parathyroid and kidney, consistent with the role of GATA3 and its interacting protein, FOG2, in the HDR syndrome. Among the cell lines, the coexpression of GATA3 and FOG2 was observed only in HEK-293 cells, and these were, therefore, selected for luciferase reporter assays to study the effect of GATA3 binding on the GCMB promoter.

The 1.2-kb DNA sequence 5' of the transcription start site $(+1)$ of the GCMB gene (Figure 5B) was cloned upstream of the Firefly luciferase gene in a pGL3 vector, and this reporter construct was transfected into HEK-293 cells. This revealed that the $1.2-\mathrm{kb}$ region upstream of the GCMB gene had regulatory activity, as it was able to induce expression of the luciferase gene approximately 20 -fold higher than the promoter-less pGL3-basic construct (Figure 5B). Furthermore, when cells were cotransfected with a GATA3 expression vector, this activity doubled $(P<0.05)$ (Figure 5B). However, when HEK-293 cells were transfected with a deletion construct that lacked the double-GATA site C, but contained the $0.9 \mathrm{~kb}$ of the GCMB upstream sequence with sites $A$ and $B$, transactivation by GATA3 was not observed (Figure 5B). These results indicate that the GATA sites A and B present in the $0.9-\mathrm{kb}$ sequence are not 


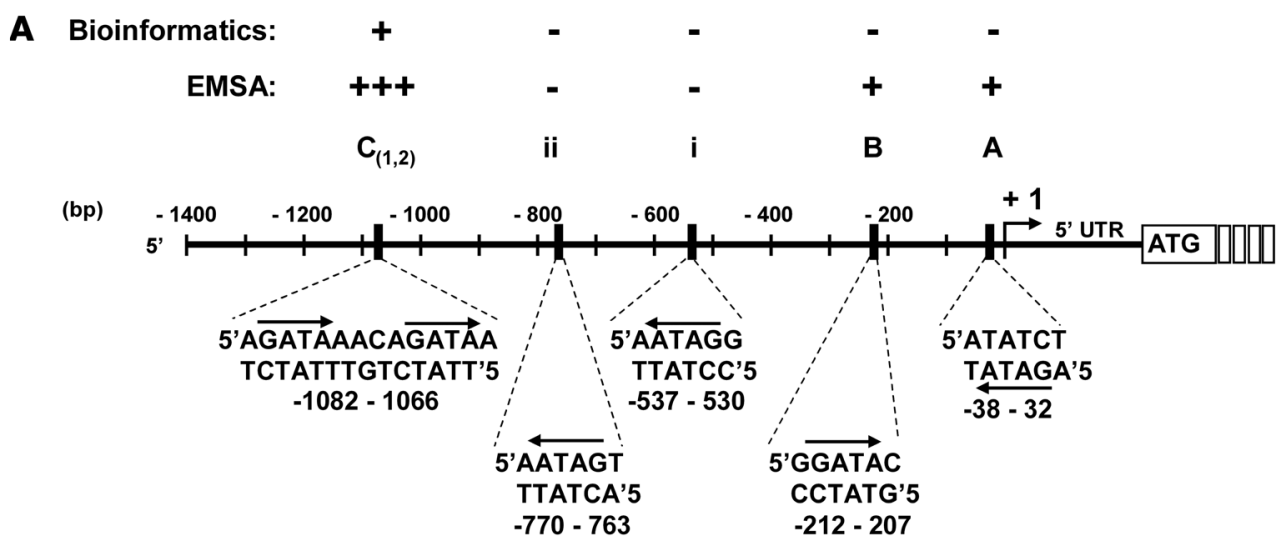

B
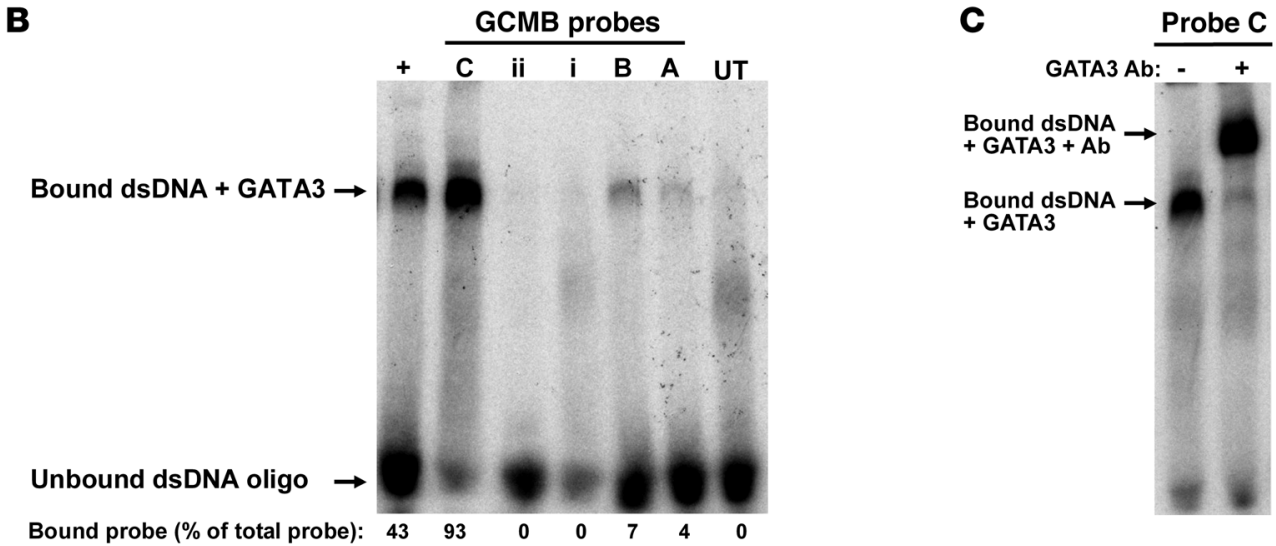

Figure 4

Analysis of DNA binding by GATA3 protein to putative GATA motifs in upstream sequence of GCMB. (A) Diagram showing location of 3 putative GATA sites (A to C) in the 1400-bp sequence upstream of the transcription site $(+1)$ of the GCMB gene. The direction of GATA motifs and their positions on the forward or reverse DNA strands are shown (arrows). Bioinformatic analysis of the 1400-bp sequence predicted only site $\mathrm{C}$ as a putative binding site of GATA3 (marked +); sites $\mathrm{A}$ and $\mathrm{B}$ and motifs $\mathrm{i}$ and ii were not predicted by this analysis (marked-). Binding by GATA3 protein to each site or motif assessed by EMSAs (B) is indicated (+++, strong; +, weak; -, absent). (B) EMSAs using nuclear extracts from COS-7 cells, transfected with the GATA3 expression vector. Binding reactions utilized a radiolabeled ( $\left.{ }^{32} \mathrm{P}\right)$ double-stranded (ds) oligonucleotide containing putative GATA site (A-C). Control binding reactions using untransfected (UT) cells and probes containing the consensus GATA binding site (+) or GATA motifs (i and ii) in the reverse orientation were performed. Densitometry revealed that more than $90 \%$ of ds oligonucleotide $\mathrm{C}$ was bound, but less than $10 \%$ of the ds oligonucleotides $A$ and $B$ were bound, and $0 \%$ of the oligonucleotides $i$ and $i$ were bound. (C) Use of an anti-GATA3 antibody in the binding reaction (+) with ds oligonucleotide containing GATA site $\mathrm{C}$ revealed a supershift, indicating that the complex was specific for the GATA3 protein.
HEK-293 cells with a reporter construct that only had a 161-bp sequence of the GCMB promoter (-1141 to $-980 \mathrm{bp}$ from transcription start site) containing site $\mathrm{C}$ and observing a 10 - to 15 fold increase in reporter activity (Figure 5B). Thus, these results indicate that transactivation by GATA3 takes place via the double-GATA site located at position -1082 to -1066 bp in the GCMB promoter. This doubleGATA site is conserved in the putative mouse $\mathrm{Gcm} 2$ promoter, suggesting that GATA3 is also likely to transactivate the $\mathrm{Gcm} 2$ promoter in a similar manner (Supplemental Figure 2).

Determining binding of GATA 3 to the GCMB promoter in vivo. ChIP experiments were performed to assess the functionality of the GATA motif at site $C$ in context of the chromatin and to test the binding by GATA3 protein to this site in vivo. In order to obtain sufficient material, we used human parathyroid adenomas, which were shown to express GATA3 and GCMB (Figure 5A). Following tissue homogenization, sonication, and crosslinking using paraformaldehyde, the presence of intact proteins was demonstrated by Western blot analysis (Figure 6, A and B). This revealed the presence of the $50-\mathrm{kDa}$ intact GATA3 protein as well as the $70 / 75-\mathrm{kDa}$ lamin A/C nuclear protein (Figure 6B). An RNApolII antibody was used to confirm binding to the promoter of the constitutively expressed GAPDH gene, thereby demonstrating functional, and this is consistent with the in vitro DNA-binding results, which revealed that these GATA sites bound GATA3 protein weakly (Figure 4, A and B). In order to assess the contribution of each of the GATA sites to the functionality of the double motifC located at position -1082 to $-1066 \mathrm{bp}$, the $\mathrm{G}$ in each GATA site was mutated to a $\mathrm{C}$, individually or in combination (Figure $5 \mathrm{~B}$ ), by site-directed mutagenesis of the $1.2-\mathrm{kb}$ reporter construct. Transfection of these mutant constructs into HEK-293 cells did not increase transactivation activity by GATA3, thereby emphasizing the importance of an intact site $\mathrm{C}$ and confirming the results from the $0.9-\mathrm{kb}$ deletional construct (Figure 5B). Mutating each GATA site individually also abolished transactivation by GATA3, indicating that an intact double-GATA motif is required. The function of this double-GATA site was independently assessed by transfecting that the ChIP assay was able to detect specific binding (Figure $6 \mathrm{C})$. PCR primers specific for the GAPDH promoter were used to show enrichment of the GAPDH PCR product (166 bp) in the fraction of chromatin immunoprecipitated with RNApolII antibody over chromatin immunoprecipitated nonspecifically with a mouse IgG. This revealed a greater abundance of the GAPDH PCR product in the RNApolII ChIP than in the IgG ChIP and both GATA3 ChIP reactions (Figure 6C). PCR primers, which flanked the double-GATA motif at site $\mathrm{C}$ in the GCMB promoter and yielded a product 160-bp long were used in a hot start PCR with DNA immunoprecipitated by 2 different GATA3 antibodies. This revealed substantially more of the $160-\mathrm{bp}$ PCR product than was observed in PCR reactions with DNA from the RNApolII ChIP, which in turn was much higher than the negative control IgG 
A
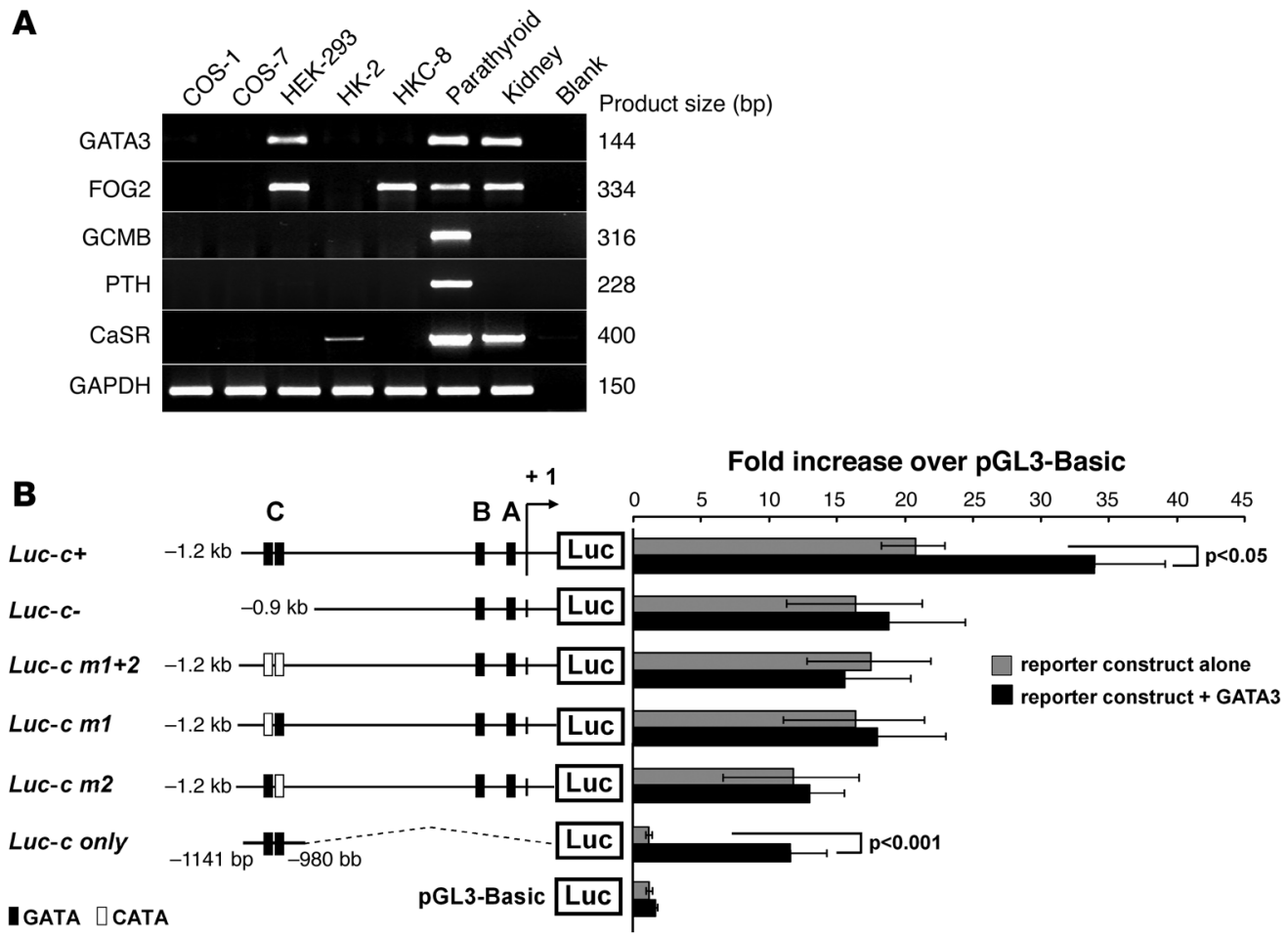

Figure 5

Luciferase reporter assays. (A) Expression of parathyroid-specific genes assessed by RT-PCR in cell lines COS-1, COS-7, HEK-293, HK-2, HKC-8, and human parathyroid adenoma and kidney. Endogenous expression of GATA3 and FOG2 was detected only in HEK-293 cells, which were therefore used in luciferase reporter assays. (B) Assessment of GATA motifs using luciferase reporter assays in HEK-293 cells. The 1.2-kb DNA sequence containing GATA motifs A-C (Figure 4) was cloned upstream of the luciferase gene in a reporter construct. HEK-293 cells were transiently cotransfected with a GATA3 expression vector or an empty vector (pcDNA3.1), lysed after 48 hours, and luminescence measured. The 1.2-kb region upstream of the GCMB gene $\left(\right.$ LuC-C $^{+}$) drove expression of the luciferase gene 20-fold higher than the promoter-less construct, pGL3-basic. Cotransfecting with a GATA3 expression vector resulted in a significant $(P<0.05)$ increase in the activity, by 2-fold. A deletion construct that lacked the double-GATA site $\left(L u C-C^{-}\right.$, Figure 3$)$ or constructs in which both GATA motifs were mutated to CATA (Luc-c $m 1+2)$, or individually (Luc-c $m 1$ and Luc-c $m 2$ ) demonstrated a loss in transactivation by GATA3. A reporter construct (Luc-c only) that had the 161-bp sequence of the GCMB promoter containing the double-GATA site placed directly upstream of the luciferase gene did not increase the transcription activity, but cotransfection with GATA3 significantly $(P<0.001)$ increased the reporter activity, by 10- to 15-fold. Mean \pm SEM of 3 independent experiments, each performed in triplicate wells, is shown.

which likely contributed to the higher mortality (Figure 1A). These findings in the Gata3 $^{+/-}$mice are consistent with the observed hypocalcemia that occurs in association with inappropriately normal or low plasma PTH concentrations in patients who have the HDR syndrome due to GATA3 haploinsufficiency. However, the hypocalcemia in HDR patients is not usually associated with a high mortality (1, 4-6), and it seems possible that other causes, which may be exacerbated by the hypocalcemia, may contribute to the higher mortality. The hypocalcemia in the Gata3+/- mice (Figure $1 \mathrm{~B})$ is mild when compared with that reported in $\mathrm{Pth}^{-/-}$ mice, which do not have a high mortality (37), and a possible explanation for the observed higher mortality in the Gata $3^{+/-}$mice (Figure 1A) is the genetic background of the Gata3 ${ }^{+/-}$mice. The Gata3 $^{+/}$- mice are on a FVB/N background, which has been reported to be associated with seizures leading to deaths (38), whereas the $\mathrm{Pth}^{-/-}$mice are on a C57BL/6 background (37), which has not been reported to be prone to seizures. It therefore seems plausible that the combined effects of the mild hypocalcemia and the susceptibility to

ChIP (Figure 6C). These observations were confirmed by quantitative SYBR Green PCR (Figure 6, D and E). This enrichment of the DNA sequences specific to the GCMB promoter and containing the double-GATA motif $\mathrm{C}$ demonstrates that GATA3 protein occupies the GCMB promoter in vivo.

\section{Discussion}

Our results demonstrate important roles for GATA3 in the embryonic development of the parathyroids and in adult parathyroid cell proliferation. Thus, Gata3 $3^{-/}$and $\mathrm{Gata}^{+/-}$embryos lacked or had smaller parathyroid-thymus primordia (Figure 2, C and D), respectively. Moreover, the parathyroids of adult $\mathrm{Gata}^{+/-}$mice did not show an increased proliferation rate or enlargement (Figure 2, $\mathrm{A}$ and $\mathrm{B}$ ) in response to hypocalcemia induced by a low calcium/vitamin D diet (Figure 1B). Indeed, this inability of the parathyroids of adult $\mathrm{Gata3}^{+/-}$mice to proliferate may help to explain the inadequate increase in plasma PTH (Figure 1C) and the subsequent failure to correct the hypocalcemia (Figure 1B), seizures in the $\mathrm{FVB} / \mathrm{N}$ strain may provide a possible explanation for the higher observed mortality in the $\mathrm{Gata3}^{+/-}$mice.

Our findings also show that the small size or absence of the parathyroids in the $\mathrm{Gata}^{+/-}$and Gata3 $3^{-/}$embryos, respectively, is due to the markedly reduced number of Gcm2-expressing cells in the third pharyngeal pouch (Figure 2, D and E). Indeed, the atrophy of the parathyroid-thymus primordia by E12.5 in Gata3 $3^{-1}$ embryos suggests that GATA3 is critical to maintaining differentiation and subsequent survival of parathyroid and thymus progenitor cells. Furthermore, our data demonstrate that GCMB is transcriptionally regulated by GATA3 (Figures 3-6). Thus, the results from EMSAs (Figure 4B), luciferase reporter assays (Figure 5B), and ChIP assays (Figure 6, C and E) revealed that GATA3 binds to the promoter of the human GCMB gene. GATA3 is specifically bound at the double-GATA motif located at position -1082 to -1066 from the transcription start site (+1) of the GCMB gene, and mutations of these GATA motifs individually or in combination resulted in a loss of 
A
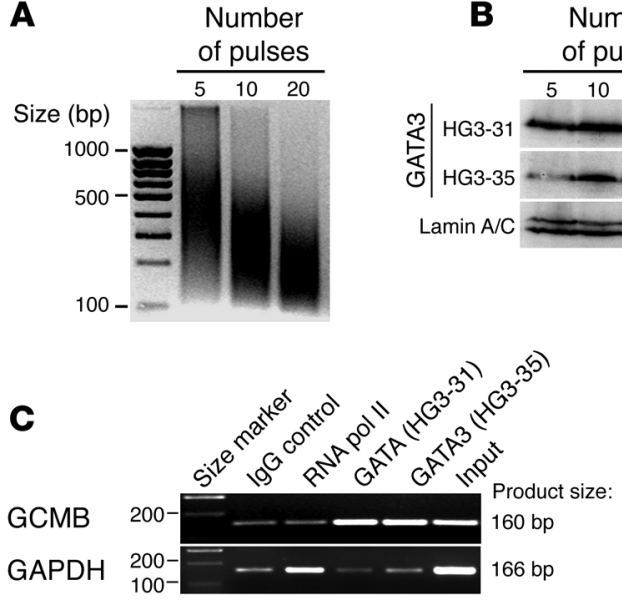

D

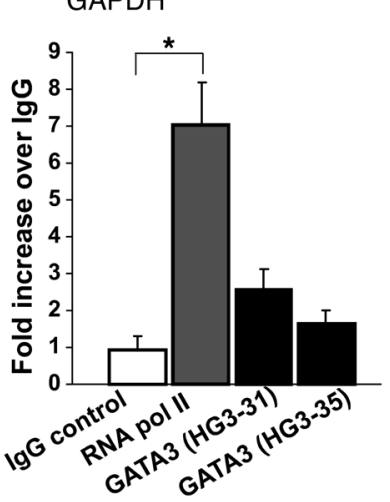

transcriptional activation in luciferase reporter assays. Importantly, this double-GATA site was shown to be occupied by GATA3 protein in vivo on the chromatin extracted from parathyroid adenoma cells, thereby indicating GATA3 has a role in regulating GCMB expression. Thus, our findings provide mechanistic insights about the regulation of this parathyroid-specific factor, which has an important role in maintaining parathyroid development (28) and cell differentiation (17).

The precise role of GATA3 in regulating GCMB expression, by either activation or repression, in normal parathyroid cells and parathyroid tumors remains to be elucidated. However, this regulation is likely to involve the CaSR, which is a G-protein-coupled receptor. A reduction in extracellular calcium causes downregulation of GCMB expression (31), enhanced parathyroid proliferation, and increased PTH expression and secretion (39), while an increase in extracellular calcium causes decreased PTH expression and secretion (40), decreased parathyroid proliferation, and upregulation of GCMB expression (31). These changes in extracellular calcium are detected by the CaSR, but the signal transduction pathways coupling CaSR to nuclear activities remain to be fully elucidated. However, activation of the CaSR by ligand binding results in activation of the MAPK pathway (41), and in human T cells, GATA3 has been shown to be a direct phosphorylation target of MAPKs $(42,43)$. For example, $T$ cell receptor-mediated activation of the Ras-ERK MAPK cascade stabilizes GATA3 protein in developing Th2 cells through the inhibition of the ubiquitin/proteasome pathway, allowing GATA3-mediated chromatin remodeling at Th2

\section{Figure 6}

ChIP assays show occupancy of the GCMB promoter by GATA3 in parathyroid tumor cells. (A) Chromatin prepared from parathyroid tumor cells using 3 chromatin sonication conditions. Sonication with 5-10 pulses produced the optimum DNA fragment size range (200$1000 \mathrm{bp}$ ) for ChIP reactions. (B) Western blot analysis of the sonicated chromatin using 2 different mouse monoclonal antibodies (HG3-31 and HG3-35) revealed the presence of the intact 50-kDa GATA3 protein. Detection of the intact $70 / 75-\mathrm{kDa}$ lamin $\mathrm{A} / \mathrm{C}$ nuclear protein was used as a control. (C) Analysis of chromatin immunoprecipitated with GATA3 antibodies, HG3-31 and HG3-35, and 2 isotype-matched control antibodies, RNApolll and IgG. Purified DNA fragments after ChIP reactions were amplified by PCR with 2 primer pairs specific for the GCMB and GAPDH promoter regions. GAPDH served as a positive control for RNApolll and a negative control for GATA3 antibodies. (D) Quantification of PCR products by SYBR Green quantitative PCR using primers specific for the GCMB and GAPDH promoter regions. The GATA3independent housekeeping gene GAPDH served as positive control for the RNApolll antibody ChIP, which demonstrated a 7-fold enrichment over IgG ChIP $(P<0.01)$. (E) Quantification of PCR products by SYBR Green quantitative PCR using primers specific for the GCMB promoter region. This confirmed the enrichment of the GCMB promoter DNA fragments immunoprecipitated with GATA3 antibodies over those immunoprecipitated with IgG and showed a 6.5- and 7.5-fold increase with HG2-31 and HG3-35 antibodies, respectively ( $\left.{ }^{*} P<0.01\right)$. Results are shown as the mean \pm SEM of 3 independent ChIP reactions. cytokine gene loci (44). This suggests the possibility that in parathyroid cells, activation of the CaSR/MAPK pathway may work to phosphorylate GATA3 and enhance its translocation into the nucleus to initiate $G C M B$ expression.

The target genes of mammalian GCMB/GCM2 are largely unknown. However, recent studies that utilized cultured primary parathyroid cells from hyperplastic glands of patients with chronic kidney disease (45) have demonstrated that downregulation of GCMB expression by infection with lentivirus expressing shRNA for GCMB resulted in downregulation of CASR expression, suggesting that one of the functions of GCMB may be to maintain high levels of CASR expression in parathyroid cells (45). These findings are supported by studies in cotransfected HEK-293, in which exogenous GCMB was able to transactivate reporter constructs containing CaSR promoter DNA sequence encompassing GCMB response elements (46). Thus, it is possible that GATA3 acts via GCMB to regulate expression of CASR, which is a G-protein-coupled receptor. This would be consistent with reports of some of the downstream target genes of GCMB that have been identified by studies in Drosophila, which have shown that $\mathrm{gcm}$ regulates expression of the repo, pointed, and loco genes $(47,48)$. The repo and pointed genes code for transcription factors that control terminal glial differentiation, while the loco gene codes for a protein with a regulator of G-protein signaling (RGS) domain. RGS proteins, which are responsible for the rapid turnoff of G-proteincoupled receptor signaling pathways (49), act via the GTPase-activating protein activity of their RGS domain, which promotes GTP 
hydrolysis by the $\alpha$ subunit of heterotrimeric G-proteins; this inactivates the G-protein and rapidly switches off G-protein-coupled receptor signaling pathways (49). A cautious extrapolation of these mechanisms to the mammalian setting suggests the possibility that GCMB may regulate expression of a mammalian RGS protein, which may act to negatively regulate signaling from the CaSR, which in turn facilitates GATA3-regulated expression of GCMB. Thus, the results of our in vitro and in vivo studies in mice deleted for Gata3, which have revealed that GCMB is regulated by GATA3, suggest new avenues to explore to elucidate this transcriptional cascade that regulates parathyroid development and extracellular calcium homeostasis.

\section{Methods}

Mouse studies. A colony of Gata3 $3^{+/-}$mice, which were congenic Gata3 $3^{+/ t a u l a c Z}$ on a FVB/N background (11), was established. All animal studies were approved by the University of Oxford Ethical Review Committee and were licensed under the Animal (Scientific Procedures) Act 1986, issued by the United Kingdom Government Home Office Department. After weaning, mice were fed a standard chow diet (Special Diets Services), which contained $1.15 \%$ calcium and $4.0 \mathrm{iU} / g$ vitamin $\mathrm{D}$; or a synthetic control diet (Dyets Inc.) containing $0.95 \% \mathrm{Ca}$ and $4.5 \mathrm{iU} / \mathrm{g}$ vitamin $\mathrm{D}_{3}$; or a matched low calcium/vitamin D diet (0.001\% Ca and $0.0 \mathrm{iU} / g$ vitamin $\left.\mathrm{D}_{3}\right)$. Food and water were allowed ad libitum. Blood was collected for analysis of plasma using an Olympus AU400 analyzer, as described (50). PTH was measured using an ELISA for mouse intact PTH (Immutopics), as described (50). The trachea, thyroid, and parathyroid were block dissected and fixed overnight in $10 \%$ neutral formalin. Embryos were collected with the day of the vaginal plug designated as E0.5 and staged by morphological criteria, which included somite number and eye and limb morphology (17).

Histology studies. Six-micron paraffin sections were stained with H\&E using standard techniques. CaSR immunohistochemistry was performed using a rabbit polyclonal anti-CaSR antibody (Abcam) and the ABC secondary detection method (Santa Cruz Biotechnology Inc.). Color reaction was developed using diaminobenzidine chromogen (DAB) solution (Vectashield), and slides were counterstained with hematoxylin. Cell proliferation was estimated using a rat anti-mouse Ki-67 antibody (Dako). Total cell number was determined by counting cell nuclei per field using the Nikon NIS Elements BR software, and the proliferation rate was expressed as Ki-67-positive cells per total cell number. Three fields from each parathyroid were counted, and the mean \pm SEM/SD determined. Whole-mount in situ hybridization using staged embryos fixed in 4\% paraformaldehyde overnight and hybridized with digoxigenin-labeled Gcm 2 RNA probe at $0.5 \mu \mathrm{g} / \mathrm{ml}$ was performed, as described (22). Alkaline phosphatase-conjugated anti-digoxigenin Fab fragments were used at 1:5,000. BM-purple (Roche) was used as a chromagen to localize the hybridized probe. Sections were counterstained with nuclear fast red.

Patients. Informed consent, as approved by a national ethical committee (London Multicentre Research Ethics Committee, United Kingdom; MREC 02/2/93), was obtained from the patients, and parathyroid adenomas were collected and frozen immediately.

5' RACE. Parathyroid adenoma total RNA was extracted using TrizoL (Invitrogen), and reverse transcription and 5' RACE-PCR were performed using $1 \mu \mathrm{g}$ of RNA and the 5'/3' RACE Kit (Roche) using universal primer mix and GCMB-specific primers (Supplemental Table 1). RACE products were cloned into TOPO vector (Invitrogen) and the DNA sequences determined as previously described (4).

In silico analysis of the $5^{\prime}$ upstream regulatory region of $G C M B$. Potential transcription factor binding sites in the promoter region of GCMB were identified by the use of the following prediction programs: TESS (www.cbil.upenn.edu/cgi-bin/tess); MatInspector (Genomatix) (www. genomatix.de); SIGNAL SCAN (www-bimas.cit.nih.gov/molbio/signal/); Match (http://www.gene-regulation.com/pub/programs.html\#match); and TRANSPLORER (www.biobase.de).

EMSAs. COS-7 cells were transfected using Lipofectamine Plus (Invitrogen) with a wild-type GATA3 construct prepared in pcDNA3.1 (Invitrogen). Forty-eight hours after transfection, the cells were harvested and nuclear extracts prepared. Five micrograms of total nuclear protein extract was used in binding reactions that utilized a ${ }^{32} \mathrm{P}$-labeled double-stranded approximately 30-bp oligonucleotide that contained a GATA motif from the endogenous GCMB promoter sequence (Figure 3) (4). The binding reactions were resolved by nondenaturing 6\% PAGE. The presence of GATA3 in the complex was demonstrated by use of an antibody against GATA3 (HG3-31; Santa Cruz Biotechnology Inc.) (5). Densitometry was carried out using the GS-710 Imaging Densitometer (Bio-Rad) and Quantity One software (Bio-Rad).

$R T-P C R$. Total RNA was extracted using Trizol (Invitrogen) from COS-7, COS-1, HEK-293, HK-2, and HKC-8 cells and used with gene-specific primer pairs (Supplemental Table 1) for GATA3, FOG2, GCMB, PTH, CaSR, and GAPDH and Superscript II reverse transcriptase (Invitrogen) (5).

GCMB reporter constructs. GCMB promoter constructs were prepared by PCR amplification of genomic DNA using a common reverse primer, GCMB-RX (5'-TCTCGAGCTGCCCAACTCGCTCGCGCTTTCC), tagged with $X$ hoI restriction site and 2 forward primers, GCMB-Luc- $\mathrm{c}^{+}$ (5'-AGGTACCCGGGAAGACAGAGTGAGACTC) and GCMB-Luc-c ${ }^{-}$ (5'-AGGTACCCTCCAGGTTCACTTTGTAGG) (Figure 3), tagged with $K p n I$ restriction sites. The PCR fragments were subcloned into pGEM-T, digested with $K p n I$ and $X h o I$, and ligated upstream of the Firefly luciferase gene in a pGL3-basic vector (Promega) digested with the same enzymes, thus generating PGL3-GCMB-Luc-c ${ }^{+}$and PGL3-GCMB-Luc-c ${ }^{-}$ constructs. Site-directed mutagenesis (QuikChange; Stratagene) was performed, as described previously (4), to generate mutations of the GATA motif in the pGL3-GCMB-Luc-c ${ }^{+}$construct (primer sequences in Supplemental Table 1). DNA sequence analysis of the plasmids was undertaken to verify the mutations. Primers GCMB-cF (5'-AAGATTTGTGTTCGGGTGGG) and GCMB-cR (5'-TGGGGAAGGAGGGAAAGGGG) were used to amplify by PCR a 161-bp product that contained a doubleGATA motif at position $-1082 \mathrm{bp}$ to $-1066 \mathrm{bp}$ from the transcription start site $(+1)$ (Figure 3 ); this PCR product was subcloned into pGEM-T, digested with KpnI and XhoI, and ligated upstream of the Firefly luciferase gene in a pGL3-basic vector (Promega).

Luciferase reporter assays. HEK-293 cells were transiently cotransfected using Lipofectamine Plus (Invitrogen) (4) with $0.4 \mu \mathrm{g}$ of a pGL3 reporter gene construct, $0.08 \mu \mathrm{g}$ of $\mathrm{pRL}$ control vector, and 0-0.5 $\mu \mathrm{g}$ of pcDNA3.1-GATA3 (wild-type or mutant) or empty pcDNA3.1 vector. Forty-eight hours after transfection, cells were lysed and assayed for luciferase activity using a Turner Biosystems luminometer and the Dual-Luciferase Reporter (DLR) assay system (Promega) (6). The Firefly luciferase activity was adjusted for Renilla luciferase activity (Firefly/Renilla ratio) and the ratios of GCMB promoter-containing constructs were corrected for those of pGL3-basic (i.e., expressed as fold increases over basic) (6). Expression of GATA3 in the transfected cells was confirmed by Western blot analysis using anti-GATA3 antibody (Santa Cruz Biotechnology Inc.).

ChIP assay. Nuclear extracts from parathyroid tumor tissue were prepared and used with the ChIP-IT kit (Active Motif). Parathyroid tumor tissue $(30-250 \mathrm{mg})$ was minced and incubated with $1 \%$ paraformaldehyde for 15 minutes, lysed in 5 mM PIPES, pH 8, $85 \mathrm{mM}$ $\mathrm{KCl}, 0.5 \% \mathrm{NP}-40$, and PMSF, homogenized using a glass Dounce homogenizer, and centrifuged at $3000 \mathrm{~g}$ for 5 minutes at $4{ }^{\circ} \mathrm{C}$ to pellet nuclei, which were resuspended in $1 \mathrm{ml}$ of shearing buffer (Active 
Motif) and sonicated using a Soniprep 150 sonicator (Sanyo) at a 6 micron amplitude (10-15 pulses of 20 seconds) to yield chromatin fragments that were of 200- to 1000-bp size, as determined by agarose gel electrophoresis. The chromatin was precleared with protein-G agarose beads (Active Motif) and immunoprecipitated with $3 \mu \mathrm{g}$ of one of the following mouse monoclonal antibodies: GATA3 (HG3-31 and HG3-35; Santa Cruz Biotechnology Inc.), RNApolII (Active Motif), and IgG (Active Motif) at $4{ }^{\circ} \mathrm{C}$ overnight. The immunoprecipitated chromatin was collected from the washed protein-G beads by adding $5 \mu \mathrm{l} 1 \mathrm{M} \mathrm{NaHCO}_{3}$ and $100 \mu \mathrm{l}$ 1\% SDS, and the covalent crosslinks between DNA and proteins were reversed by overnight incubation at $65^{\circ} \mathrm{C}$ with $4 \mu \mathrm{l}, 5 \mathrm{M} \mathrm{NaCl}$. The samples was further incubated with $10 \mu \mathrm{g}$ RNase A at $37^{\circ} \mathrm{C}$ for 30 minutes, followed by $10 \mu \mathrm{g}$ proteinase $\mathrm{K}$ at $42^{\circ} \mathrm{C}$ for 1 hour. The immunoprecipitated DNA was purified using mini-spin columns (Active Motif) and eluted in $50 \mu \mathrm{l}$ of $\mathrm{dH}_{2} \mathrm{O}$. PCR was performed using JumpStart Taq DNA polymerase (Sigma-Aldrich) at $26-35$ cycles at $57^{\circ} \mathrm{C}$ (annealing temperature) with GCMB-specific primers (GCMB-cF, -1121 to $-1140 \mathrm{bp}, 5$ '-AAGATTTGTGTTCGGGTGGG; and GCMB-cR, -980 to -1000 bp, 5'-TGGGGAAGGAGGGAAAGGGG; Figure 3). Positive control GAPDH primers (GAPDH-For 5'-TACTAGCGGTTTTACGGGCG; GAPDH-Rev 5'-TCGAACAGGAGGAGCAGAGAGCGA) were used as controls to detect enrichment by the RNApolII antibody. The PCR products were resolved by $2 \%$ agarose gel electrophoresis. To quantify enrichment, ABI 7900HT-based real-time PCR analysis (Applied Biosystems) was performed with SYBR Green I PCR Master Mix (Invitrogen), and the fold increase over DNA immunoprecipitated nonspecifically with
IgG was calculated using the formula $2^{(\Delta \Delta C t)}$, where $C t=$ the cycle number at which the fixed threshold is crossed; $\Delta C t=C t$ (immunoprecipitated sample) minus $C t$ (input); and $\Delta \Delta C t=\Delta C t$ (IgG) minus $\Delta C t$ (ChIP Ab).

Statistics. Statistical analysis was performed using SPSS version 14.0 and Microsoft Excel. Significance of differences was assessed by unpaired, 2 -tailed Student's $t$ test, Mann-Whitney $U$ test, or $\chi^{2}$ test. $P<0.05$ was considered significant.

\section{Acknowledgments}

We thank the Medical Research Council (MRC), UK (grant number 69825289 [2004]), for support. I.V. Grigorieva, S. Mirczuk, and K.U. Gaynor are or were MRC-funded PhD students; A. Ali was an MRC Clinical Training Fellow; J.M. Stacey received a short-term European Molecular Biology Organization (EMBO) fellowship; and M.J. Stechman was a Kidney Research UK Clinical Training Fellow. We are grateful to P. Christie and J. Jeyabalan for assistance.

Received for publication December 14, 2009, and accepted in revised form March 31, 2010.

Address correspondence to: Rajesh V. Thakker, Academic Endocrine Unit, Nuffield Department of Medicine, Oxford Centre for Diabetes, Endocrinology and Metabolism (OCDEM), Churchill Hospital, University of Oxford, Headington, Oxford, OX3 7LJ, United Kingdom. Phone: 44.1865.857501; Fax: 44.1865.857502; E-mail: rajesh.thakker@ndm.ox.ac.uk.
1. Van Esch H, et al. GATA3 haplo-insufficiency causes human HDR syndrome. Nature. 2000; 406(6794):419-422.

2. Simon MC. Gotta have GATA. Nat Genet. 1995 ; 11(1):9-11.

3. Fox AH, Liew C, Holmes M, Kowalski K, Mackay J, Crossley M. Transcriptional cofactors of the FOG family interact with GATA proteins by means of multiple zinc fingers. EMBOJ. 1999;18(10):2812-2822.

4. Nesbit MA, et al. Characterization of GATA3 mutations in the hypoparathyroidism, deafness, and renal dysplasia (HDR) syndrome.J Biol Chem. 2004;279(21):22624-22634.

5. Ali A, et al. Functional characterization of GATA3 mutations causing the hypoparathyroidism-deafnessrenal (HDR) dysplasia syndrome: insight into mechanisms of DNA binding by the GATA3 transcription factor. Hum Mol Genet. 2007;16(3):265-275.

6. Gaynor KU, et al. A missense GATA3 mutation, Thr272Ile, causes the hypoparathyroidism, deafness, and renal dysplasia syndrome. J Clin Endocrinol Metab. 2009;94(10):3897-3904.

7. Pandolfi PP, et al. Targeted disruption of the GATA3 gene causes severe abnormalities in the nervous system and in fetal liver haematopoiesis. Nat Genet. 1995;11(1):40-44.

8. Grote D, et al. Gata3 acts downstream of betacatenin signaling to prevent ectopic metanephric kidney induction. PLoS Genet. 2008;4(12):e1000316.

9. Grote D, Souabni A, Busslinger M, Bouchard M. Pax 2/8-regulated Gata 3 expression is necessary for morphogenesis and guidance of the nephric duct in the developing kidney. Development. 2006; 133(1):53-61.

10. Milo M, et al. Genomic analysis of the function of the transcription factor gata 3 during development of the mammalian inner ear. PLoS ONE. 2009;4(9):e7144.

11. van Doorninck JH, et al. GATA-3 is involved in the development of serotonergic neurons in the caudal raphe nuclei. J Neurosci. 1999;19(12):RC12.

12. van Looij MA, et al. Characteristics of hearing loss in HDR (hypoparathyroidism, sensorineural deafness, renal dysplasia) syndrome. Audiol Neurootol.
2006;11(6):373-379.

13. van Looij MA, et al. GATA3 haploinsufficiency causes a rapid deterioration of distortion product otoacoustic emissions (DPOAEs) in mice. Neurobiol Dis. 2005;20(3):890-897.

14. van der Wees J, et al. Hearing loss following Gata3 haploinsufficiency is caused by cochlear disorder. Neurobiol Dis. 2004;16(1):169-178.

15. Manley NR, Capecchi MR. Hox group 3 paralogs regulate the development and migration of the thymus, thyroid, and parathyroid glands. Dev Biol. 1998;195(1):1-15.

16. Su D, Ellis S, Napier A, Lee K, Manley NR. Hoxa3 and pax1 regulate epithelial cell death and proliferation during thymus and parathyroid organogenesis. Dev Biol. 2001;236(2):316-329.

17. Liu Z, Yu S, Manley NR. Gcm2 is required for the differentiation and survival of parathyroid precursor cells in the parathyroid/thymus primordia. Dev Biol. 2007;305(1):333-346.

18. Bowl MR, et al. An interstitial deletion-insertion involving chromosomes $2 \mathrm{p} 25.3$ and Xq27.1, near SOX3, causes X-linked recessive hypoparathyroidism. J Clin Invest. 2005;115(10):2822-2831.

19. Zhang $Z$, et al. Tbx1 expression in pharyngeal epithelia is necessary for pharyngeal arch artery development. Development. 2005;132(23):5307-5315.

20. McCarron DA, Muther RS, Lenfesty B, Bennett WM. Parathyroid function in persistent hyperparathyroidism: relationship to gland size. Kidney Int. 1982;22(6):662-670.

21. Panda DK, et al. Inactivation of the 25 -hydroxyvitamin D 1alpha-hydroxylase and vitamin D receptor demonstrates independent and interdependent effects of calcium and vitamin D on skeletal and mineral homeostasis. J Biol Chem. 2004; 279(16):16754-16766.

22. Gordon J, Bennett AR, Blackburn CC, Manley NR. Gcm 2 and Foxn 1 mark early parathyroid- and thymus-specific domains in the developing third pharyngeal pouch. Mech Dev. 2001;103(1-2):141-143.

23. Moore-Scott BA, Manley NR. Differential expression of Sonic hedgehog along the anterior-poste- rior axis regulates patterning of pharyngeal pouch endoderm and pharyngeal endoderm-derived organs. Dev Biol. 2005;278(2):323-335.

24. Patel SR, Gordon J, Mahbub F, Blackburn CC, Manley NR. Bmp4 and Noggin expression during early thymus and parathyroid organogenesis. Gene Expr Patterns. 2006;6(8):794-799.

25. Blackburn CC, Manley NR, Palmer DB, Boyd RL, Anderson G, Ritter MA. One for all and all for one: thymic epithelial stem cells and regeneration. Trends Immunol. 2002;23(8):391-395.

26. Blackburn CC, Manley NR. Developing a new paradigm for thymus organogenesis. Nat Rev Immunol. 2004;4(4):278-289.

27. Griffith AV, et al. Increased thymus- and decreased parathyroid-fated organ domains in Splotch mutant embryos. Dev Biol. 2009;327(1):216-227.

28. Gunther T, et al. Genetic ablation of parathyroid glands reveals another source of parathyroid hormone. Nature. 2000;406(6792):199-203.

29. Ding C, Buckingham B, Levine MA. Familial isolated hypoparathyroidism caused by a mutation in the gene for the transcription factor GCMB. J Clin Invest. 2001;108(8):1215-1220.

30. Thomee C, et al. GCMB mutation in familial isolated hypoparathyroidism with residual secretion of parathyroid hormone. J Clin Endocrinol Metab. 2005;90(5):2487-2492.

31. Kebebew E, Peng M, Wong MG, Ginzinger D, Duh QY, Clark OH. GCMB gene, a master regulator of parathyroid gland development, expression, and regulation in hyperparathyroidism. Surgery. 2004;136(6):1261-1266.

32. Norton JA. Surgical management of hyperparathyroidism. In: DeGroot LJ, Jameson JL, eds. Endocrinology. $5^{\text {th }}$ ed. Philadelphia, PA: Elsevier Saunders; 2006:1583-1594.

33. Kanemura $Y$, et al. Isolation and expression analysis of a novel human homologue of the Drosophila glial cells missing $(\mathrm{gcm})$ gene. FEBS Lett. 1999;442(2-3):151-156.

34. Maret A, Ding C, Kornfield SL, Levine MA. Analysis of the GCM2 gene in isolated hypoparathyroidism: 
a molecular and biochemical study. J Clin Endocrinol Metab. 2008;93(4):1426-1432.

35. Sakai Y, Nakagawa R, Sato R, Maeda M. Selection of DNA binding sites for human transcriptional regulator GATA-6. Biochem Biophys Res Commun. 1998;250(3):682-688.

36. Mackay JP, Kowalski K, Fox AH, Czolij R, King $\mathrm{GF}$, Crossley M. Involvement of the N-finger in the self-association of GATA-1. J Biol Chem. 1998; 273(46):30560-30567.

37. Miao D, et al. Skeletal abnormalities in Pth-null mice are influenced by dietary calcium. Endocrinology. 2004;145(4):2046-2053.

38. Mahler JF, Stokes W, Mann PC, Takaoka M, Maronpot RR. Spontaneous lesions in aging FVB/ $\mathrm{N}$ mice. Toxicol Pathol. 1996;24(6):710-716.

39. Chen RA, Goodman WG. Role of the calcium-sensing receptor in parathyroid gland physiology. $A m J$ Physiol Renal Physiol. 2004;286(6):F1005-F1011.

40. Kifor O, Kifor I, Brown EM. Signal transduction in the parathyroid. Curr Opin Nephrol Hypertens.
2002;11(4):397-402.

41. Brown EM, MacLeod RJ. Extracellular calcium sensing and extracellular calcium signaling. Physiol Rev. 2001;81(1):239-297.

42. Yu YL, et al. MAPK-mediated phosphorylation of GATA-1 promotes Bcl-XL expression and cell survival. J Biol Chem. 2005;280(33):29533-29542.

43. Maneechotesuwan K, et al. Regulation of Th2 cytokine genes by p38 MAPK-mediated phosphorylation of GATA-3. J Immunol. 2007; 178(4):2491-2498.

44. Yamashita M, et al. Ras-ERK MAPK cascade regulates GATA3 stability and Th2 differentiation through ubiquitin-proteasome pathway. J Biol Chem. 2005;280(33):29409-29419.

45. Mizobuchi M, Ritter CS, Krits I, Slatopolsky E, Sicard G, Brown AJ. Calcium-sensing receptor expression is regulated by glial cells missing- 2 in human parathyroid cells. J Bone Miner Res. 2009; 24(7):1173-1179.

46. Canaff L, Zhou X, Mosesova I, Cole DE, Hendy GN
Glial cells missing-2 (GCM2) transactivates the calcium-sensing receptor gene: effect of a dominantnegative GCM2 mutant associated with autosomal dominant hypoparathyroidism. Hum Mutat. 2009;30(1):85-92.

47. Granderath S, Bunse I, Klambt C. gcm and pointed synergistically control glial transcription of the Drosophila gene loco. Mech Dev. 2000;91(1-2):197-208.

48. Granderath S, Stollewerk A, Greig S, Goodman CS O'Kane CJ, Klambt C. loco encodes an RGS protein required for Drosophila glial differentiation. Development. 1999;126(8):1781-1791.

49. De Vries L, Zheng B, Fischer T, Elenko E, Farquhar MG. The regulator of $\mathrm{G}$ protein signaling family. Annu Rev Pharmacol Toxicol. 2000;40:235-271.

50. Harding B, et al. Multiple Endocrine Neoplasia Type 1 (MEN1) knockout mice develop parathyroid, pancreatic, pituitary and adrenal tumours with hypercalcaemia, hypophosphataemia and hypercorticosteronaemia. Endocr Relat Cancer. 2009; 16(4):1313-1327. 